\title{
Cross-Sensory Modulation of Primary Sensory Cortex Is Developmentally Regulated by Early Sensory Experience
}

\author{
Ayan Ghoshal, Andrew Tomarken, and Ford Ebner \\ Department of Psychology, Vanderbilt University, Nashville, Tennessee 37240
}

The presence of cross-sensory influences on neuronal responses in primary sensory cortex has been observed previously using several different methods. To test this idea in rat $S 1$ barrel cortex, we hypothesized that auditory stimuli combined with whisker stimulation ("cross-sensory" stimuli) may modify response levels to whisker stimulation. Since the brain has been shown to have a remarkable capacity to be modified by early postnatal sensory activity, manipulating postnatal sensory experiences would be predicted to alter the degree of cross-sensory interactions. To test these ideas, we raised rats with or without whisker deprivation and with or without postnatal exposure to repeated auditory clicks. We recorded extracellular responses under urethane anesthesia from barrel cortex neurons in response to principal whisker stimulation alone, to auditory click stimulation alone, or to a cross-sensory stimulus. The responses were compared statistically across different stimulus conditions and across different rearing groups. Barrel neurons did not generate action potentials in response to auditory click stimuli alone in any rearing group. However, in cross-sensory stimulus conditions the response magnitude was facilitated in the $0-15 \mathrm{~ms}$ post-whisker-stimulus epoch in all rearing conditions, whereas modulation of response magnitude in a later 15-30 ms post-whisker-stimulus epoch was significantly different in each rearing condition. The most significant cross-sensory effect occurred in rats that were simultaneously whisker deprived and click reared. We conclude that there is a modulatory type of cross-sensory auditory influence on normal $\mathrm{S} 1$ barrel cortex, which can be enhanced by early postnatal experiences.

\section{Introduction}

The convergence of information from more than one sensory modality greatly improves the sensitivity of an animal's perceptually guided behavior (Stein and Meredith, 1990; Burnett et al., 2004). Psychophysical experiments have shown that multisensory interactions also modulate perception in humans. For example, in illusions, such as the "parchment-skin illusion," somesthetic perceptions for rough and smooth surfaces are dramatically modulated by concurrent sounds (Jousmäki and Hari, 1998; Guest et al., 2002). Similarly, there is evidence for somesthetic-visual and audio-visual interactions. However, in this report we concentrate on somesthetic-auditory interactions.

Primary sensory cortex has been considered devoid of multisensory interactions. In the last 2 decades, however, a strong case has emerged for the notion that multisensory modulation of sensory responses is a common feature of both primary $(\mathrm{V} 1, \mathrm{~S} 1, \mathrm{~A} 1)$ and secondary (V2, S2, auditory belt) sensory areas. Such phenomena are often referred to as "cross-sensory" or "cross-modal" interactions (for review, see Foxe and Schroeder, 2005; Ghazanfar and Schroeder, 2006; Kayser and Logothetis, 2007). Physiological evidence supports the concept of a linkage among all combinations of visual, auditory, and somatic sensory inputs to

Received 0ct. 22, 2010; revised Dec. 7, 2010; accepted Dec. 10, 2010.

This work was supported by the Center for Integrative \& Cognitive Neuroscience, the Vanderbilt Vision Research Center, the Vanderbilt Kennedy Center, and a Vanderbilt University Discovery Grant. We thank Dipanwita Ghose for assistance with the histology, and Dr. Daniel Polley for technical assistance while characterizing the auditory stimuli.

Correspondence should be addressed to Dr. Ford Ebner, Department of Psychology, Wilson Hall, 11121st Avenue South, Vanderbilt University, Nashville TN 37240. E-mail: ford.ebner@vanderbilt.edu.

DOI:10.1523/JNEUROSCI.5547-10.2011

Copyright $\odot 2011$ the authors $\quad 0270-6474 / 11 / 312526-11 \$ 15.00 / 0$ cortex. Somatic and visual stimuli can strongly activate single and multiunits in A1 and the posterior auditory belt area when the stimuli are relevant to an overtrained task (Brosch et al., 2005). Lakatos et al. (2007) found event-related potential responses in the supragranular layers of macaque A1 cortex in response to somatic sensory stimuli, suggesting a modulatory as opposed to a driving type of cross-sensory influence in the primary auditory area, even when overtraining was not a variable. Moreover, bimodal stimuli, composed of paired somatic sensory and auditory stimuli, led to a supralinear multisensory facilitation both for field potentials and multiunit activity in all layers of A1 (Lakatos et al., 2007). Only a few studies have investigated multisensory properties in S1 cortex. In monkeys performing a haptic discrimination task, Zhou and Fuster $(2000,2004)$ located neurons in Brodmann's areas $3 \mathrm{a}, 3 \mathrm{~b}, 1$, and 2 that could be activated by visual or auditory cues when linked to the haptic task. Dynamic crosssensory plasticity is also well documented where plasticity refers to the ability of active sensory modalities to recruit or strongly modulate neurons in a deprived area of cortex (for review, see Bavelier and Neville, 2002). Sensory deprivation of a particular modality during development diminishes the response magnitude of the deprived neurons (Wiesel and Hubel, 1965; Simons and Land, 1987; Stern et al., 2001; Popescu and Ebner, 2010) and disrupts neuronal synchrony (Ghoshal et al., 2009) in primary cortical areas representing that modality. The net effect is that "deprived" cortical neurons become less responsive to natural stimuli. However, deprived cortex, in turn, has a remarkable capacity to respond to cross-sensory stimulation or reorganize in response to the suppression of the natural sensory inputs (for review, see Collignon et al., 2009; Kral, 2007). 
In the present study, we investigated whether neuronal responses in rat $\mathrm{S} 1$ barrel cortex could be modulated or driven by an auditory click stimulus after early somatic sensory deprivation. It has been shown before that rearing rats with auditory clicks in the background can reorganize auditory cortex (Zhang et al., 2002), and thus, a further goal was to determine whether increased auditory stimulation during whisker deprivation would augment the cross-sensory activation of barrel cortex. Our results show that whisker-driven responses in rat $\mathrm{S} 1$ barrel cortex are modulated by auditory inputs after normal rearing, and that this influence is significantly altered following atypical early sensory experience.

\section{Materials and Methods}

All of the experiments in this report were approved by the Vanderbilt University Institutional Animal Care and Use Committee, performed in an Association for Assessment and Accreditation of Laboratory Animal Care-approved animal facility, and were in accordance with the guidelines of the National Institutes of Health and the Society for Neuroscience.

Animal groups. The experimental design consisted of four rearing groups using 17 male (10) and female (7) Long-Evans rats (250-350 g, 2-3 months at the time of recording). The newborn pups were raised either (1) with a continuous $1 \mathrm{~Hz}$ auditory click stimulus in addition to normal laboratory noises [click reared $(\mathrm{CR}+)$ ] or (2) with normal laboratory noise only $(\mathrm{CR}-)$. Half of the animals in each of these groups were reared with bilateral whisker trimming [i.e., whiskers bilaterally deprived (WBD)] and half without any sensory deprivation [sham trimming (i.e., control $[\mathrm{CON}])]$ to generate four rearing conditions: $\mathrm{N}_{\mathrm{CON} / \mathrm{CR}-}=5$; $\mathrm{N}_{\mathrm{CON} / \mathrm{CR}+}=4 ; \mathrm{N}_{\mathrm{WBD} / \mathrm{CR}-}=4 ; \mathrm{N}_{\mathrm{WBD} / \mathrm{CR}+}=4$, as illustrated in Figure $1 \mathrm{~A}$.

Bilateral whisker deprivation. Two groups of rats were bilaterally sensory deprived (WBD) by trimming all whiskers from both sides of the face to the level of the fur, for a period of $60 \mathrm{~d}$ beginning on the day of birth [postnatal day (PND) 0-60]. Two groups of animals were handled but not trimmed during the same period to serve as $\mathrm{CON}$ groups. Whiskers were trimmed every day until PND 30 (when the rate of the whisker growth was most rapid) and every other day thereafter. During preweaning whisker trimming, the whisker-trimmed pups were caged with their control littermates and nursed by their dam. After weaning, same-sex animals were housed in groups of three to five animals per cage, depending on their size. Five days before recording sessions, trimming was discontinued and the whiskers were allowed to regrow to a length sufficient to stimulate each whisker without moving the small facial hairs $(\sim 5$ $\mathrm{mm}$ ). This $5 \mathrm{~d}$ period of whisker regrowth was the only recovery period in these studies.

Auditory click rearing. One group of WBD and one group of CON rats were reared from PND 0 to 60 with a repeated click stimulus delivered at $1 \mathrm{~Hz}$ continuously through a speaker kept above the rat cages [click duration: $10 \mathrm{~ms}$; amplitude: $75 \mathrm{~dB}$ sound pressure level (spl) at $20 \mathrm{~mm}$ from the speaker]. The click stimulus was generated by a square wave pulse generator (Grass Technologies) and consisted of an envelope of frequencies (range: $1 \mathrm{~Hz}$ to $50 \mathrm{kHz}$ ).

Surgery and recording. Over a period of days from 1 week to 1 month after the end of the rearing period, each rat was anesthetized with urethane $(1.5 \mathrm{~g} / \mathrm{kg}, 30 \%$ aqueous solution, i.p.), and mounted in a head holder that allowed free access to the whiskers and did not require the use of ear bars (Narishige). A craniotomy was made from 4 to $8 \mathrm{~mm}$ lateral to the midline and from 0 to $5 \mathrm{~mm}$ posterior to bregma to expose the left barrel field cortex (BFC). Body temperature was maintained at $37^{\circ} \mathrm{C}$ with a feedback-actuated heating pad (Harvard Apparatus). Supplementary injections ( $10 \%$ of the initial dose) were given as needed to maintain the anesthesia at stage III-3 (Friedberg et al., 1999). After making a small opening in the dura mater, microelectrodes were advanced in columnar penetrations perpendicular to the cortical surface. Contact with the pia was identified visually through an operation microscope. Commercial quartz glass-insulated, platinum/iridium microelectrodes were used having 2-6 $\mathrm{M} \Omega$ resistance (Thomas Recording). The electrodes were advanced into the brain using an Eckhorn microdrive system (Thomas
Recording). Analog waveform signals were amplified by Thomas preamps and collected by a Plexon Multichannel Acquisition Processor system in which the waveforms were digitized at $40 \mathrm{kHz}$. Multiunit activity was viewed on-line using Sort Client software (Plexon) and stored for off-line analysis. The electrode was first advanced to layer IV (LIV) of the BFC where receptive fields were mapped manually. The whisker was identified on-line that evoked the largest amplitude response in a multiunit post-stimulus time histogram (PSTH), which was designated as the "principal whisker" (PW) (PeriEvent Client, Plexon). Multiunit activity was collected from layers II and III (LII/III; depth, 100-450 $\mu \mathrm{m}$ ) and LIV (depth, 450-800 $\mu \mathrm{m}$ ) of the BFC (Li et al., 2005).

The multiunit spike stream collected on-line was sorted off-line to isolate single-neuron waveforms, and the magnitude and spike timing of responses to each of the three stimulus conditions were compared.

Electrodes were advanced or retracted in $100 \mu \mathrm{m}$ intervals to minimize the probability of recording twice from the same units. Lesions were made by passing DC current ( $1 \mu \mathrm{A}$ for $2 \mathrm{~min}$ ) at depths of $600 \mu \mathrm{m}$ in each penetration, and their location was later verified anatomically using cytochrome oxidase-stained tangential sections through the BFC. However, the lesions were too variable in size to precisely locate the recording location to either a barrel or a septum.

Whisker and auditory stimulation during recording. Before recording, the whiskers were trimmed at $5 \mathrm{~mm}$ beyond the fur in all groups to keep the whisker length uniform. A piezoelectric bimetal wafer was used to deliver 100 stimuli to the whiskers in a caudal direction $(1 \mathrm{~Hz}, 600 \mu \mathrm{m}$ amplitude, $4 \mathrm{~ms}$ duration, $2 \mathrm{~ms}$ rise time). The piezoelectric wafer was actuated by a "custom" waveform programmed in a digital stimulator (DS8000, WPI), which in turn was controlled by a Spike 2 script program (CED). The auditory stimulus was generated by a second channel on the DS8000, which was also controlled by a Spike 2 script to activate a speaker (Kenwood) (20-20,000 Hz response). The speaker was positioned 20 $\mathrm{mm}$ away from the right ear of the rat. Auditory stimuli were delivered at $1 \mathrm{~Hz}(75 \mathrm{~dB}$ spl, $10 \mathrm{~ms}$ duration square wave either alone or $10 \mathrm{~ms}$ before a whisker stimulus). Five different sets of stimuli were presented in each recording location always in the same sequence (see Fig. $1 B$ ), as follows: (1) contralateral PW stimulation alone (W1) before the CS stimuli (100 trials); (2) contralateral auditory stimulation alone before the CS stimuli (A1) (100 trials); (3) contralateral auditory stimulus preceding whisker stimulation by $10 \mathrm{~ms}$ (CS) (100 trials); (4) contralateral auditory stimulation after CS stimuli (A2) (100 trials); and (5) contralateral principal whisker stimulation $5 \mathrm{~min}$ after CS stimuli (W2) (100 trials).

We locked the interstimulus interval (ISI) between the auditory click and the whisker stimulus at $10 \mathrm{~ms}$ and did not test other possible ISIs due to technical limitations. That is, some of the effects, especially in the $\mathrm{WBD} / \mathrm{CR}+$ rats, were longer lasting, making it difficult to interpret whether the effect observed for a particular ISI was characteristic for that temporal interval or due to the residual effect of the previous ISI tested.

Since under our conditions neither A1 nor A2 evoked a significant spiking response in LII/III or LIV barrel neurons in any neurons in any of the four rearing groups, these stimulus conditions were not analyzed further for magnitude differences.

Data analysis. Principal component analysis and template matching were used in Offline Sorter (Plexon) for sorting spike waveforms to separate single units from the mulitunit stream. Typically, two to three units per electrode could be isolated for further analysis from each electrode recording position. PSTHs were constructed from waveform time stamps for each cell in response to the different stimulus conditions using a custom NEX script (provided by Dr. Alexander Kirillov, NEX Technologies, Littleton, MA). Raster plots generated by the NEX software were used to display individual cell trial-by-trial spike frequencies and latencies. PSTHs and raster plots for W1, CS, and W2 conditions were constructed with the onset of the whisker stimulus as the reference event under each condition, whereas, those for A1 and A2 were constructed with the onset of the square wave input to the speaker as the reference event. Significant responses to each stimulus condition were determined using a $99 \%$ confidence interval calculated by the NEX software. The magnitude of response for each neuron was calculated as spike counts in the first and second $15 \mathrm{~ms}$ poststimulus epoch $(0-15$ and $15-30 \mathrm{~ms}$ post-whisker-stimulus). The magnitude for spontaneous firing of neu- 
rons was calculated as the total spike count in a time window of the last $500 \mathrm{~ms}$ before each successive stimulus during the block of 100 trials. The response modulation index (RMI) for each rearing group was calculated as the ratio of magnitude $(\mathrm{CS}) /$ magnitude $(\mathrm{CS}+\mathrm{W} 1)$ and represented a quantitative measure for the amount of cross-sensory influence. A high RMI value thus indicates a relative facilitation of responses in the CS stimulus, whereas, a low RMI value represents suppression. These values were compared statistically between rearing groups and/or across stimulus conditions within a rearing group. Population PSTHs were constructed by averaging the responses of all neurons from the same group and producing a graphic display (after smoothing the graphs with 3 Gaussian filters) using NEX software and a custom MATLAB script.

Significant increases in response magnitude of individual neurons in either the $0-15$ or the $15-30$ ms epoch were identified when the average of 100 trials in the CS condition exceeded the sum of the mean plus two times the SE of $100 \mathrm{~W} 1$ trials. A decrease in response magnitude for individual neurons was identified for either of the epochs when the trial average in the CS condition was lower than the value of the mean plus $2^{\star} \mathrm{SE}$ for $100 \mathrm{~W} 1$ trials. This analysis was performed to evaluate the percentage of individual neurons in each rearing group that showed either the facilitative or suppressive crosssensory modulations described in the results.

Statistical analyses. We performed analyses testing as follows: (1) the overall effects of postnatal rearing and stimulus variables on spike counts; and (2) a series of designed comparisons addressing more focused questions. Spike counts summed across two poststimulus time periods ( $0-15$ and $15-30 \mathrm{~ms}$ ) served as the dependent measures. To test effects, we used a generalized linear mixed-effects model (GLMM), which is an extension of the generalized linear model (GLM). GLMs specifying that the data have either a Poisson or negative binomial distribution are commonly used to analyze counts (Hardin and Hilbe, 2007). In turn, GLMMs are an extension of GLMs that are appropriate when independence of observations is violated due to repeated measures or other factors that reflect clustering of observations (Fitzmaurice et al., 2004). In the present study, there were several sources of clustering that needed to be accounted for: the correlated responses of a given neuron recorded across the three stimulation conditions; the shared correlation among all the neurons of a given rat; and the correlated responses of neurons recorded from the same cortical layer within a given animal (a more local factor that might serve to heighten the correlation among neurons at the same depth). Such structures can be modeled by the specification of random effects that allow for estimation of the shared variance among all observations within a given source of clustering. The random effects specification for stimulation condition within rat included an additional scaling parameter that corrected for potential overdispersion (i.e., underestimation of SEs) in the data. By these means, we were able to ensure that hypothesis tests were more valid for the effects of primary interest (i.e., the effects of rearing group and stimulus condition) (Fitzmaurice et al., 2004). All random effects were assumed to be normally distributed. Because the numbers of counts per epoch were generally high, and because in the limit the Poisson distribution approaches a normal distribution, an alternative approach would have been to use a linear or generalized linear mixed-effects model that specified a normal distribution of residuals. In fact, the GLMM approach that we used and these alternative approaches yielded results that were highly similar and conclusions that were identical.

Our initial models tested for the main effects and interactions among rearing condition $(\mathrm{CON} / \mathrm{CR}-, \mathrm{CON} / \mathrm{CR}+, \mathrm{WBD} / \mathrm{CR}-, \mathrm{WBD} / \mathrm{CR}+$ ), stimulus condition (W1, CS, W2), and cortical layer (II/III or IV). Main effects refer to the overall effects of a given factor averaging across all relevant neurons. Thus, for example, the main effect of rearing condition tested the global null hypothesis that the means of the four rearing conditions computed by averaging across all neurons and stimulus conditions were equal to one another. Interactions tested whether the effects of a given factor were conditional upon the specific levels of other factors. To generate a suitable number of observations, we aggregated cortical layer into two groups, LII/III (100-450 $\mu \mathrm{m}$ in depth) and LIV (450-800 $\mu \mathrm{m}$ in depth), to create the cortical layer factor.

Analyses were conducted using SAS PROC GLIMMIX software, version 9.1.3 of the SAS system for Windows (SAS Institute; SAS and all other SAS Institute products or service names are registered trademarks of SAS Institute) (Littell et al., 2006). Consistent with the count nature of the dependent variables, both the $0-15$ and $15-30$ ms models specified a Poisson distribution for the dependent variables. In addition to the global tests of interest, we tested several a priori contrasts of prime interest. Specifically, we compared the counts of the W1 condition to those of the CS and W2 conditions within each of the four rearing groups. To control familywise type 1 error rates, we set the critical $\alpha$ level per group at $0.05 / 4=0.0125$ and then used a stepdown Bonferroni procedure to evaluate the two contrasts within each group. These sets of contrasts were performed separately for the $0-15$ and $15-30 \mathrm{~ms}$ time windows. Using a similar Bonferroni procedure, we compared the W1 and CS conditions within each of the two cortical layer groupings for each rearing group. More exploratory analyses are also described in the Results, such as whether excitatory versus inhibitory status of neurons moderated the effects. The rationale behind the statistical approach is explained in detail in the supplemental Methods (available at www.jneurosci.org as supplemental material).

\section{Results}

Somatosensory and auditory interactions in normal, whisker-deprived, and/or click-reared rat barrel cortex

In the present experiments, we tested the hypothesis that auditory stimuli can influence the responses of the barrel cortex neurons under normal conditions and under conditions of whisker deprivation coupled with auditory-enhanced rearing. Three of five stimulus conditions (W1, CS, and W2) (Fig. 1B) elicited significant responses from the barrel cortex neurons under all four rearing conditions (Fig. 1). The responses from W1, CS, and W2 were divided into early and later post-stimulus time epochs of 0-15 and 15-30 ms because deprivation and click rearing produced consistent response modifications in these two time windows. Changes in response magnitude were analyzed in each epoch to compare the three stimulus conditions. In addition, most of the neurons recorded in each rearing group were stimulated with auditory click stimulation alone (A1, A2) (Fig. 1B) both before and after the CS stimulus. However, the click stimulation alone failed to produce detectable changes in firing rate from barrel cortex neurons in any of the rearing groups (data not shown).

Raster plots (Fig. $1 C-F$ ) show the responses of representative single neurons from each of the four rearing groups in response to W1 (whisker before CS), CS (auditory-whisker, cross-sensory stimulus), and W2 (whisker after CS) stimuli. The plots show that there is a modulation in the response of a neuron under CS stimulus conditions when compared with $\mathrm{W} 1$. The responses in the $\mathrm{W} 2$ condition are similar to the $\mathrm{W} 1$ response pattern. The response modulations in a single cortical neuron (Fig. $1 C-F$ ) closely resemble the population effects discussed below (Fig. 2). For example, in Figure $1 F$ the CS stimulus produced an increase in response magnitude in a single neuron in $\mathrm{WBD} / \mathrm{CR}+$ rats in the $0-15 \mathrm{~ms}$ epoch, followed by a striking suppression of response in the 15-30 ms time window. This effect shows up clearly in the population of neurons recorded from $\mathrm{WBD} / \mathrm{CR}+$ rats (Fig. 2D).

\section{Omnibus analyses of the magnitude of cross-sensory responses}

To analyze group effects by rearing, stimulus and cortical layer, we performed GLMM analyses on spike counts occurring during the poststimulus windows of $0-15$ and $15-30 \mathrm{~ms}$ [rearing (CON/ $\mathrm{CR}-, \mathrm{CON} / \mathrm{CR}+, \mathrm{WBD} / \mathrm{CR}-, \mathrm{WBD} / \mathrm{CR}+) \times$ stimulus condition $(\mathrm{W} 1 / \mathrm{CS} / \mathrm{W} 2) \times$ layer (II-III, IV)] (Table 1). To account for sources of nonindependence, we specified random effects for rat, cortical layer within rat, and stimulus condition within neuron. 
A

\begin{tabular}{|c|c|c|}
\hline CONDITIONS & $\begin{array}{c}\text { UN-CLICKED } \\
\text { Rearing }\end{array}$ & $\begin{array}{c}\text { CLICKED } \\
\text { Rearing }\end{array}$ \\
\hline $\begin{array}{c}\text { Control } \\
\text { (CON) }\end{array}$ & & \\
\hline $\begin{array}{c}\text { Bilaterally whisker } \\
\text { Deprived (WBD) } \\
\text { PND 0-60 }\end{array}$ & $\begin{array}{c}\text { CON/CR- } \\
\text { WBD/CR- }\end{array}$ & CON/CR+ + \\
\hline
\end{tabular}

B

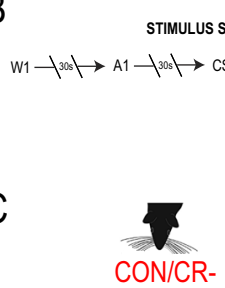

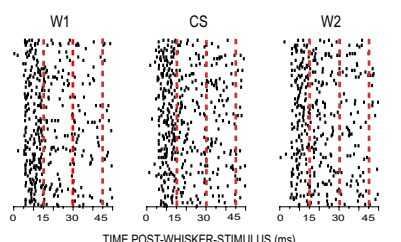

E
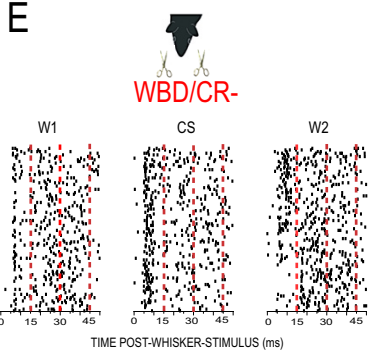

$\mathrm{F}$
A

A
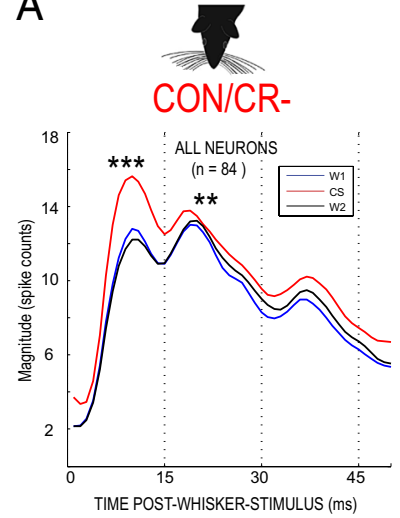

$\mathrm{C}$
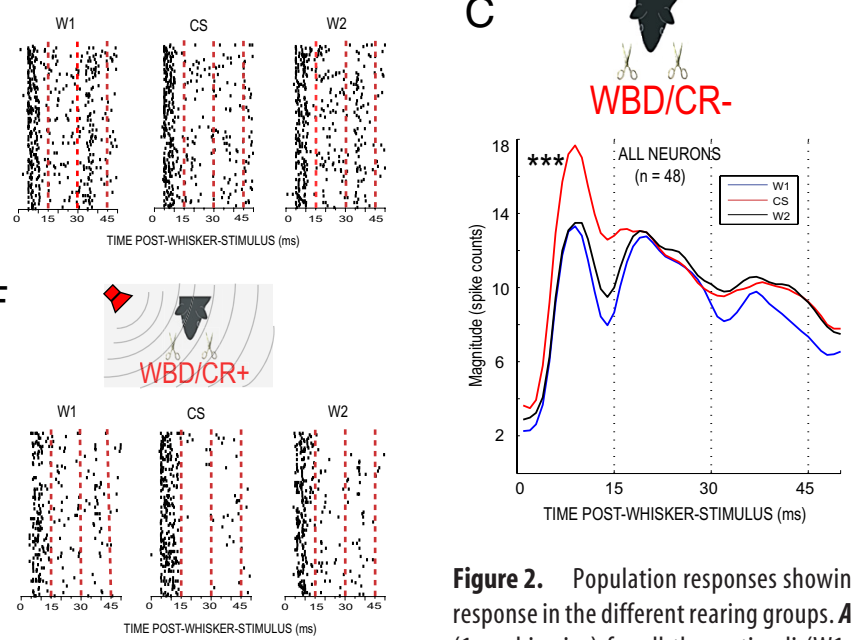

Figure 1. Experimental design and single cell examples demonstrating cross-sensory interactions in barrel cortex. $A$, Experimental design showing the four rearing conditions from birth to PND 60. CON or WBD rats were raised either with normal laboratory noise ( $C R-$ ) or reared with $1 \mathrm{~Hz}$ auditory clicks $(C R+)$. $B$, Sequence of stimulation used to test single cell responses in barrel cortex of all animals. Neuronal responses were analyzed to whisker-only stimuli before (W1) and after (W2) a CS stimulus. W2 was always performed 5 min after the last CS stimulus. Click alone (A1 and A2) always produced a null response. $\mathbf{C}-\boldsymbol{F}$, Raster plots of typical single neurons from each of the rearing groups showing responses to the $W 1, C S$, and W2 stimuli over a 50 ms post-whisker-stimulus time period. Zero milliseconds on the $x$-axis is the onset of the whisker stimulus in each stimulus condition. Vertical, red, dashed lines in the plots demark the first three $15 \mathrm{~ms}$ post-whisker-stimulus epochs. The increase in the number of spikes in the $0-15$ ms epoch of the $C S$ stimulus condition was significant in all the rearing groups when compared with W1. The responses in the $15-30 \mathrm{~ms}$ time window were more variable across the rearing groups, with the $\mathrm{WBD} / \mathrm{CR}+(\boldsymbol{F})$ showing a dramatic suppression of response in the $C S$ stimulus condition. The W2 stimulus, delivered 5 min after the CS condition appear to be not different from the $W 1$ responses.

The GLMM performed on the $0-15$ ms window yielded a highly significant main effect for stimulus condition $(p<0.0001)$ due to a highly significant increase in neural responses elicited by the CS stimulus in all four rearing conditions. No other effects were statistically significant (all $p$ values $>0.09$ ). Subsequent multiple comparisons (Fisher least significant difference) (Levin et al., 1994) among the three types of stimulus indicated that the response magnitude was higher in the CS condition relative to both the W1 $(p<0.0001)$ and the W2 conditions $(p<0.0001)$. The $\mathrm{W} 1$ and $\mathrm{W} 2$ conditions were not significantly different $(p>$ 0.70) (Fig. 2).

The omnibus GLMM analysis conducted on the $15-30 \mathrm{~ms}$ epoch showed a different result. We observed significant main
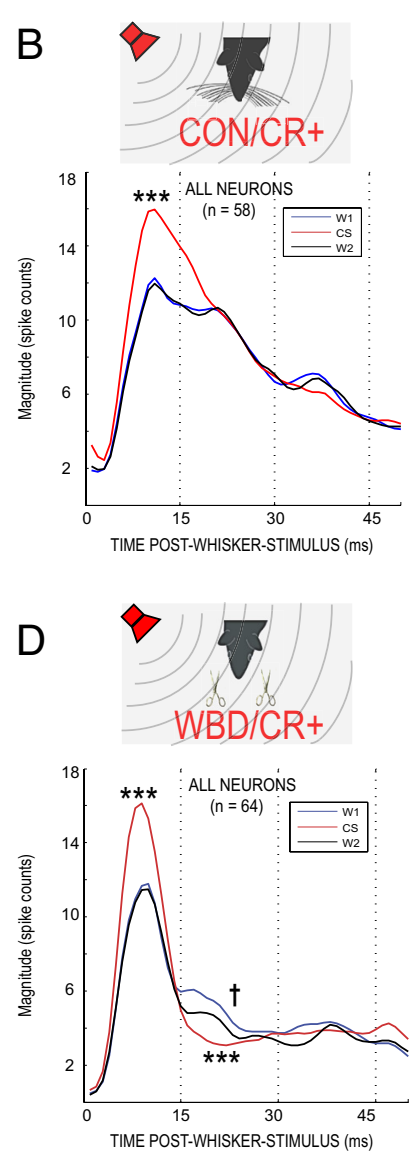

Figure 2. Population responses showing the cross-sensory effect on the magnitude of response in the different rearing groups. $\boldsymbol{A}-\boldsymbol{D}$, Population post-stimulus-time histograms (1 ms bin size) for all three stimuli (W1, CS, and W2) under the four different rearing conditions. The onset of the whisker stimulus is time 0 . The PSTHs were smoothed using NEX software with Gaussian filters with a filter bin width of 3 and divided into $15 \mathrm{~ms}$ post-whisker-stimulus time windows (dashed black vertical lines). The response magnitude to the $C S$ stimulus was significantly increased in the $0-15 \mathrm{~ms}$ epoch for all four rearing groups, whereas in the $15-30 \mathrm{~ms}$ epoch there was significant $\mathrm{CS}$ facilitation in CON/CR - rats $(\boldsymbol{A})$ and a significant CS suppression in the WBD/CR + rats (D). No significant changes in response magnitude to the $C S$ stimulus were observed in the $15-30 \mathrm{~ms}$ in $\mathrm{CON} / \mathrm{CR}+$ rats $(\boldsymbol{B})$ and $\mathrm{WBD} / \mathrm{CR}$ - rats $(\boldsymbol{C})$. The response to the W2 stimulus was not significantly different from that to the W1 stimulus for any rearing groups at any time, except in the WBD/CR + rats $(\boldsymbol{D})$ where the responses to the $W 2$ stimulus in the $15-30 \mathrm{~ms}$ epoch were significantly lower. See Table 1 for mean values and SEMs for each epoch. ${ }^{* * *} p<0.0001$, CS compared with W1; ${ }^{* *} p<0.01$, CS compared with W1; ${ }^{\dagger} p<0.05$, W2 compared with W1.

effects for rearing $(p<0.05)$, stimulus condition $(p<0.0001)$, and layer $(p<0.05)$, which were moderated by a highly significant rearing $\times$ stimulation interaction $(p<0.0001)$ (Fig. 2$)$ and a significant three-way rearing $\times$ stimulation $\times$ layer interaction $(p<0.04)$ (supplemental Fig. 1, available at www.jneurosci.org as supplemental material). Thus, unlike the short latency epoch, the effects of stimulation on the 15-30 ms time window were conditional on both rearing condition and layer. Further analyses also showed that these cross-sensory effects, especially the ones observed in the 15-30 ms epoch were somewhat dependent upon the responsiveness of the neurons, with the low responsive neurons exhibiting the cross-sensory modulations in the $15-30 \mathrm{~ms}$ epoch, more often than the high responsive neurons (Fig. 3). How each rearing group was influenced by cross-sensory stimuli is presented in detail in the next section. 
Table 1. Total spike counts for 100 trials in the $0-15$ and 15-30 ms post-whisker stimulus epoch for each stimulus condition and rearing group

\begin{tabular}{|c|c|c|c|c|c|c|}
\hline \multirow[b]{2}{*}{ Rearing groups } & \multicolumn{3}{|l|}{$0-15$ ms epoch } & \multicolumn{3}{|l|}{ 15-30 ms epoch } \\
\hline & W1 & CS & W2 & W1 & CS & W2 \\
\hline $\mathrm{CON} / \mathrm{CR}-$ & $132.99(22.97)$ & $166.75(28.66)$ & $129.86(22.44)$ & $166.85(39.28)$ & $181.52(42.71)$ & $172.81(40.68)$ \\
\hline $\mathrm{CON} / \mathrm{CR}+$ & $117.40(20.70)$ & $148.09(25.93)$ & $115.92(20.45)$ & $123.83(29.59)$ & $120.73(28.86)$ & $123.03(29.4)$ \\
\hline WBD/CR- & $114.41(32.91)$ & $154.12(31.94)$ & $115.61(24.14)$ & $158.60(43.73)$ & $159.91(44.08)$ & $165.42(45.58)$ \\
\hline WBD/CR+ & $100.36(18.11)$ & $121.01(21.71)$ & 99.68 (17.99) & 76.65 (18.75) & $48.98(12.13)$ & $67.16(16.49)$ \\
\hline
\end{tabular}

Values are given as means (SEMs) for 100 trials in the $0-15$ and $15-30$ ms post-whisker-stimulus epoch for each stimulus condition and rearing group. The means shown are estimated population marginal means adjusted for all fixed effects (i.e., main effects and interactions) in the model (Searle et al. 1980). The means displayed are in the scale of raw counts and are thus exponentiated versions of the estimates directly yielded by the generalized mixed-model analysis. SEMs were estimated using the delta method (Littell et al. 2006).

\section{Cross-sensory interactions in normal $(\mathrm{CON} / \mathrm{CR}-)$ rats}

To test for cross-sensory interactions, we compared the response magnitude in the $0-15$ and $15-30$ ms epochs after the W1 stimulus to that of the other two stimulus conditions (CS and W2) within each of the four rearing groups.

Normal rats reared with intact whiskers in a normal laboratory noise environment showed significant cross-sensory interaction in S1 barrel cortex. Population PSTHs, constructed by averaging the responses of 84 neurons to W1, CS, and W2 stimuli showed a significant modulation of neuronal response to the CS stimulus in normal animals (CON/CR-) (Fig. 2A). The magnitude of spike counts following the CS stimulus was significantly greater than after the W1 stimulus during the $0-15$ ms poststimulus epoch $(p<0.0001)$, whereas the W1 spike counts were not significantly different from the W2 spike counts $(p>0.05)$. Over $65 \%$ of all neurons showed an increased response to CS stimulation in the $0-15 \mathrm{~ms}$ epoch. During the 15-30 ms epoch, the response magnitude to CS stimuli was also significantly greater than that to $\mathrm{W} 1$ in normal animals $(p<0.005)$, whereas the magnitude of response to $\mathrm{W} 2$ stimuli remained unaltered from the response to $\mathrm{W} 1(p>0.05)$ (Fig. $2 A)$. When tested individually, $51 \%$ of all neurons showed the increase in magnitude to CS stimuli in the 15-30 ms epoch.

\section{Cross-sensory interactions in $\mathrm{CON} / \mathrm{CR}+$ rats}

Control rats with intact whiskers were also reared with environmental click stimuli (CON/CR+) to show the effect of click rearing by itself. Recordings from 58 neurons of this group from LII/III and LIV were pooled together to construct the population PSTHs to W1, CS, and W2 stimuli (Fig. 2 B). Similar to the other rearing groups, the magnitude of the neuronal response to the CS stimulus was significantly higher in the $0-15$ ms epoch when compared with the response to the W1 stimulus condition $(p<$ 0.0001 ) when the responses to W1 were not significantly different from those to W2 ( $p>0.05)$. This facilitative effect with the CS stimulus was observed in $81 \%$ of neurons in the CON/CR+ group. The click rearing in normal animals failed to show any difference in response magnitude in the $15-30 \mathrm{~ms}$ epoch. Thus, the responses under CS and W2 stimulus conditions were not significantly different from that under W1 (W1 vs CS, $p>0.05$; $\mathrm{W} 1$ vs $\mathrm{W} 2, p>0.05)$. However, when individual neurons were analyzed $46 \%$ actually showed a decrease in magnitude in this epoch with the CS stimulus.

\section{Cross-sensory interactions in $\mathrm{WBD} / \mathrm{CR}$ - rats}

Rats whisker trimmed bilaterally, but not click reared, served as a control for sensory deprivation alone. This group also showed significant modulation of barrel cortex responses to the CS stimulus that were evident only in the initial $0-15$ ms poststimulus epoch. This result can be seen in the population PSTHs constructed from responses of 48 neurons combined from both LII/ III and LIV of barrel cortex (Fig. 2C). The CS stimulus in WBD/CR- animals caused a significant increase in the magnitude of the neuronal responses in the $0-15$ ms epoch when compared with the response to the W1 stimulus $(p<0.0001)$, without a significant $\mathrm{W} 1-\mathrm{W} 2$ difference in this $0-15 \mathrm{~ms}$ epoch $(p>0.05)$. This increase with CS stimulus was found in $81 \%$ of individual neurons in this group, showing that the sensory deprivation alone produced an enhanced response to the cross-sensory stimulus. Like the CON/CR+ rats, the magnitude of the longer latency 15-30 ms epoch was not significantly different among the three stimulus conditions (W1 vs $\mathrm{CS}, p>0.05$; or W1 vs W2, $p>0.05$ ).

\section{Cross-sensory interactions in $\mathrm{WBD} / \mathrm{CR}+$ rats}

Rats with their whiskers trimmed bilaterally and reared with environmental auditory clicks from PND 0 to 60 showed the greatest modulation of response magnitude to the CS stimulus. All of the neurons recorded from the WBD/CR + barrel cortex $(n=64)$ were pooled to construct population PSTHs for the different stimulus conditions (Fig. 2D). For the $0-15 \mathrm{~ms}$ epoch, there was a significant increase in magnitude of the response to the CS stimulus when compared with that of the W1 stimulus $(p<$ 0.0001 ); whereas, the magnitude of response to W2 stimulus remained unaltered in this epoch compared with the response to W1 $(p>0.05)$. This increase in magnitude to the CS stimulus was found in $69 \%$ of all neurons.

In contrast, in the 15-30 ms epoch, there was a highly significant decrease in the magnitude of response to CS stimulation when compared with the $\mathrm{W} 1$ responses $(p<0.0001)$ with $75 \%$ of neurons in the population showing this effect. Moreover, when the whisker alone was stimulated again (W2), the response remained significantly less than that to $\mathrm{W} 1(p<$ 0.012 ) in the $15-30 \mathrm{~ms}$ post-whisker stimulus epoch. Thus, in the WBD/CR+ animals the CS stimuli increased the short latency whisker response and strongly suppressed the longer latency whisker response.

\section{Dependence of cross-sensory interactions on the responsiveness of neurons}

All neurons recorded were subdivided into "high" and "low" responsive groups in each rearing condition. For a given rearing condition, if the total spike count of a neuron (0-30 ms poststimulus epoch) in response to whisker stimulus alone (W1) was higher than the median of the total spike counts of all neurons, then it was considered a high responsive neuron and vice versa. Figure 3 illustrates how the CS stimuli affected the magnitude of 0-15 and 15-30 ms epochs of the high and low responsive neurons in each rearing condition.

In the $0-15 \mathrm{~ms}$ epoch, the facilitation of response to the CS stimulus was significant in both responsive groups for all rearing conditions, except for the high responsive neurons of WBD/CR+ rats $(p>0.05)$ (Fig. $3 D)$.

In the 15-30 ms epoch of CON/CR - rats, only the low responsive neurons showed a significant facilitation $(p<0.01)$ 
A

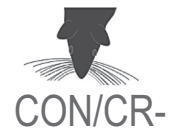

$B$

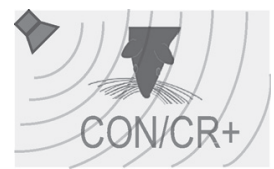

C

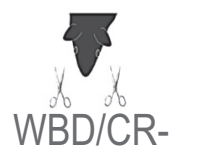

D

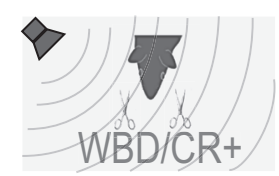

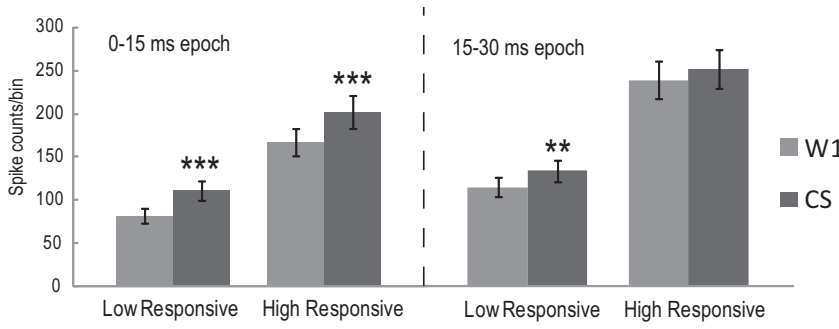
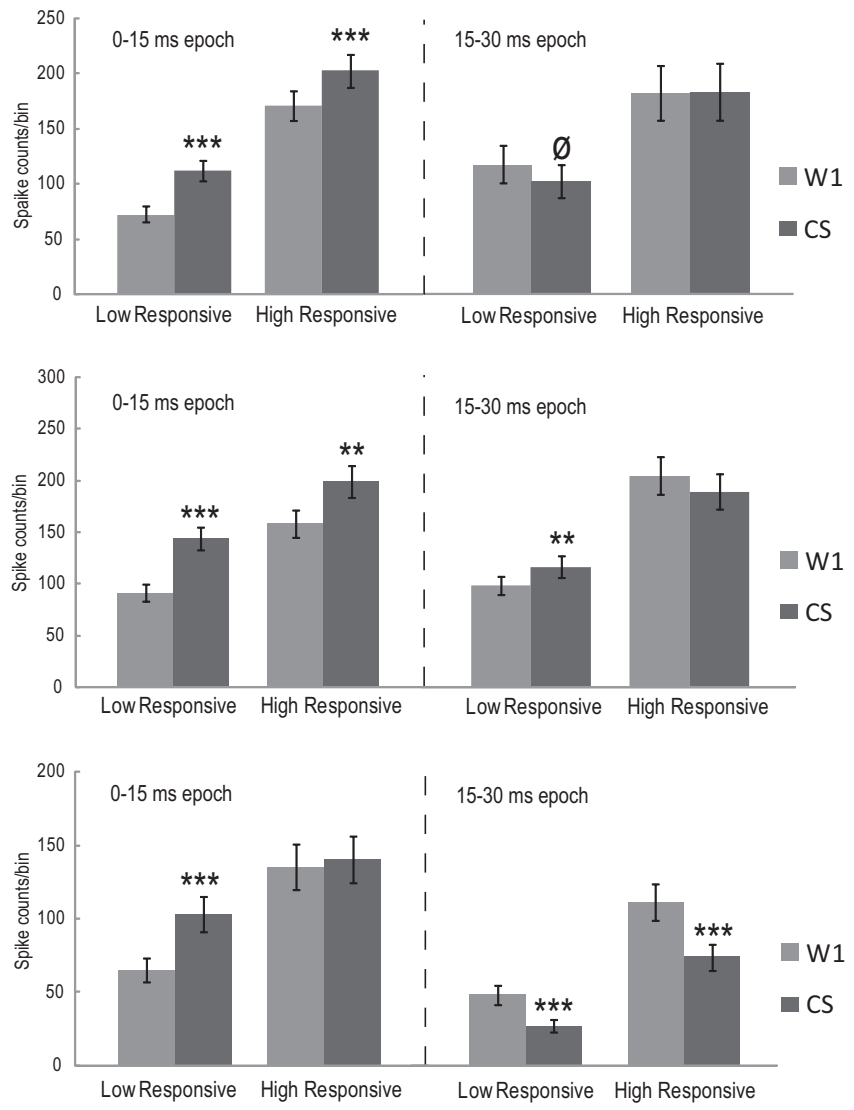

Figure 3. Population means of responses in each rearing group showing the dependence of cross-sensory effect on neuronal responsiveness. Neurons for each rearing group were divided into two groups depending on whether their mean response in the $30 \mathrm{~ms}$ post-whisker-stimulus period was below (low responsive) or above (high responsive) the median of responses of all the neurons in that rearing group. $\boldsymbol{A}-\boldsymbol{D}$, Bar graphs for total spike counts for each such neuronal group in either the $0-15 \mathrm{~ms}$ (left column) and 15-30 ms post-whisker stimulus epoch (right column) are displayed for CON/CR $(\boldsymbol{A}), \mathrm{CON} / \mathrm{CR}+(\boldsymbol{B}), \mathrm{WBD} / \mathrm{CR}-(\boldsymbol{C})$, and WBD/CR + (D) rats. Responses for W1 (light gray) and CS (dark gray) stimulus conditions are considered and compared statistically using a stepdown Bonferroni procedure. The response facilitation in the $0-15 \mathrm{~ms}$ epoch after the $\mathrm{CS}$ stimulus is observed in both responsive types for all rearing groups except the high responsive group of the $W B D / C R+$ rats. For the $15-30$ ms epoch only low responsive neurons of $C O N / C R$ - and $W B D / C R-$ rats showed significant facilitation of response $(A, C)$; whereas, with click rearing only $(C O N / C R+)$ there is a strong trend for suppression of neuronal response in low responsive cells $(\boldsymbol{B})$. Simultaneous click rearing and whisker deprivation (WBD/CR +) leads to highly significant response suppression in both the low and high responsive neurons in the $15-30 \mathrm{~ms}$ poststimulus epoch (D). ${ }^{* * *} p<0.001 ;{ }^{* *} p<0.01 ;{ }^{\emptyset} p=0.03$. Error bars indicate SEMs.

(Fig. 3A). For the CON/CR+ rats, the responses in this epoch were not significantly different in response to the CS stimulus for both the responsive groups ( $p>0.0125$ ), but the low responsive neurons showed a trend toward response suppression following the CS stimulus ( $p=0.03$; stepdown Bonferroni critical $p=0.0125$ ) (Fig. $3 B$ ). In the WBD/CR - rats, the magnitude in the 15-30 ms epoch of all neurons was not significantly different in the CS stimulus condition (Fig. 2C). However, when classified according to responsiveness, the low responsive neurons showed a significant facilitation of response $(p<0.01)$ (Fig. $3 C)$. For the $\mathrm{WBD} / \mathrm{CR}+$ rats, there was a significant response suppression for both the high and low responsive neurons in the 15-30 ms epoch $(p<0.001)$ (Fig. $3 D)$. A stepdown Bonferroni procedure was applied to adjust the $p$ values for multiple tests (see Materials and Methods).

\section{Dependence of cross-sensory interaction on laminar position of neurons}

We further subdivided the pool of neurons according to their location in LII/III or LIV to determine whether the crosssensory interactions observed in the 4 rearing groups were different in different cortical layers. Neurons that were recorded from depths ranging from 100 to $450 \mu \mathrm{m}$ were considered to be in the supragranular LII/III, whereas neurons recorded from 450 to $800 \mu \mathrm{m}$ were considered to be in LIV (Li et al., 2005). Average responses of the neurons from each layer were used to construct the population PSTHs for W1, CS, and W2 stimuli in each rearing group, as displayed in supplemental Figure 1 (available at www. jneurosci.org as supplemental material). In the $0-15 \mathrm{~ms}$ time window, the increase in magnitude of response under the CS stimulus condition was consistent across all layers. That is, both LII/III and LIV neurons of all rearing conditions showed a significant increase in spike counts in the 0-15 ms epoch when stimulated with the CS stimulus compared with the response following W1 stimulus (CON/CR-: LII/ III, $n=38, p<0.0001$; and LIV, $n=46$, $p<0.0001$; CON/CR+: LII/III, $n=27$, $p<0.0001$; and LIV, $n=31, p<0.01$; WBD/CR-: LII/III, $n=18, p<0.001$; and LIV, $n=30, p<0.0001 ; \mathrm{WBD} / \mathrm{CR}+$ : LII/III, $n=27, p<0.05$; and LIV, $n=37$, $p<0.001)$.

There was a subtle relationship between the laminar positions of neurons and the cross-sensory interactions in the 15-30 ms epoch observed in certain rearing groups (supplemental Fig. 1, available at www.jneurosci.org as supplemental material). In the CON/CR - rats, the increase in magnitude in the 15-30 ms under the CS stimulus condition was significant only in LIV $(p<0.02)$ but not in LII/III ( $p>0.05$ ). The lack of any evident cross-sensory interaction in this epoch (i.e., differences in the response magnitude between $\mathrm{W} 1$ and $\mathrm{CS}$ conditions for $\mathrm{WBD} / \mathrm{CR}-$ and $\mathrm{CON} / \mathrm{CR}+$ ) was consistent in both LII/III and LIV $(p>0.05)$. For WBD/ $\mathrm{CR}+$ rats, the decreased response magnitude to the CS stimulus was highly significant in both LII/III $(p<0.0001)$ and LIV $(p<$ 0.0001 ) when compared with that of the W1 response. A stepdown Bonferroni procedure was applied to adjust the $p$ values for multiple tests (see Materials and Methods). Thus, the laminar position of neurons had only a minor effect on the cross-sensory interactions reported above. 
Dependence of cross-sensory interaction on putative excitatory and inhibitory neurons

To determine whether a particular neuron type is more susceptible to the cross-sensory interactions observed in rat barrel cortex, we divided the entire pool of neurons from each rearing group into regular spiking units (RSUs) and fast spiking units (FSUs). A neuron was designated an RSU or an FSU based on their baseline-tobaseline waveform duration (RSU, $>750 \mu \mathrm{s}$; FSU, <750 $\mu \mathrm{s}$ ). For each rearing group, population PSTHs were constructed averaging the responses of RSUs and FSUs to W1, CS, and W2 stimulus conditions (supplemental Fig. 2, available at www.jneurosci.org as supplemental material). For the $0-15 \mathrm{~ms}$ epoch, the increase in magnitude of response to CS stimulus over the responses to $\mathrm{W} 1$ was significant for RSUs in all the rearing groups $[\mathrm{CON} / \mathrm{CR}-(n=53), p<0.0001 ; \mathrm{CON} /$ $\mathrm{CR}+,(n=31), p<0.0001 ; \mathrm{WBD} / \mathrm{CR}-$, $(n=27), p<0.0001 ; \mathrm{WBD} / \mathrm{CR}+,(n=38)$, $p<0.001]$. For FSUs, the increase was only significant for CON/CR $-(n=28$; $p<$ $0.01), \mathrm{WBD} / \mathrm{CR}-(n=21 ; p<0.0001)$, and $\mathrm{CON} / \mathrm{CR}+(n=27 ; p<0.01)$ rats, but not for $\mathrm{WBD} / \mathrm{CR}+$ rats $(n=26 ; p>0.05)$.

For the $15-30 \mathrm{~ms}$ epoch in the CON/ $\mathrm{CR}$ - rats, the increase in magnitude of responses with CS stimulus over that of W1 stimulus was significant only for RSUs ( $p<$ 0.05 ) but not for FSUs ( $p>0.05$ ). However, in the $\mathrm{WBD} / \mathrm{CR}+$ rats the decrease in magnitude with CS stimulus compared with the W1 responses in this epoch was significant in both RSUs $(p<0.0001)$ and FSUs $(p<$ $0.0001)$. No significant differences between $\mathrm{W} 1$ and CS stimulus responses were observed in this epoch for WBD/CR - RSUs $(p>0.05)$ or FSUs $(p>0.05)$ and CON/ $\mathrm{CR}+\mathrm{RSUs}(p>0.05)$ and FSUs $(p>$ $0.05)$. As above, a stepdown Bonferroni procedure was applied to adjust the $p$ values for multiple tests (see Materials and Methods).

\section{The effect of the cross-sensory stimuli on spontaneous activity}

The magnitudes of spontaneous firing rates were calculated using the total spike counts in a $500 \mathrm{~ms}$ time window from 500 to $1000 \mathrm{~ms}$ post-whisker-stimulus and was compared across stimulus conditions within each group. Both non-click-reared groups (CON/CRand $\mathrm{WBD} / \mathrm{CR}-$ ) showed a significant increase in spontaneous activity in this time window $(p<0.01)$ under the CS stimulus condition, but both click-reared groups $(\mathrm{CON} / \mathrm{CR}+$ and $\mathrm{WBD} / \mathrm{CR}+$ ) showed no change in spontaneous activity $(p>0.05)$ during the $C S$ stimulus condition (Table 2).

\section{Between-group comparisons of}

\section{cross-sensory interaction using the RMI}

To compare whether the auditory-touch interactions observed in our experimental rearing group $\mathrm{WBD} / \mathrm{CR}+$ were significantly different from the other control groups, we statistically compared the RMI of the $0-15$ and 15-30 ms epochs across different groups. For each neuron assessed, the RMI was computed by the following ratio: magnitude $(\mathrm{CS}) /$ magnitude $(\mathrm{CS}+\mathrm{W} 1)$, separately for each epoch. We used this index for two reasons. First, it is easily interpretable because it is bounded between 0 and 1 (i.e., it assesses the proportion of the total number of counts across the CS and W1 conditions that generated by an alternative GLMM.
Table 2. Total spike counts for 100 trials during the relatively spontaneous periods ( 500 to 1000 ms post-whisker stimulus) for each stimulus condition and each rearing group

\begin{tabular}{llr}
\hline & $500-1000$ ms spontaneous epoch \\
\cline { 2 - 3 } Rearing groups & W1 & \multicolumn{1}{l}{ CS } \\
\hline CON/CR- & $1033.61(365.45)$ & $1147.32(405.38)$ \\
CON/CR+ & $474.63(172.24)$ & $486.73(176.54)$ \\
WBD/CR- & $983.87(404.50)$ & $1153.52(473.73)$ \\
WBD/CR+ & $171.92(65.59)$ & $171.9(65.53)$ \\
\hline
\end{tabular}

Values are given as the mean (SEM) of total spike counts for 100 trials during the relatively spontaneous periods (500 to 1000 ms post-whisker-stimulus) for each stimulus condition and each rearing group. Themeans shown are estimated population marginal means adjusted for all fixed effects (i.e., main effects and interactions) in the model (Searle et al. 1980). The means displayed are in the scale of raw counts and are thus exponentiated versions of the estimates directly yielded by the generalized mixed-model analysis. SEMs were estimated using the delta method (Littell et al. 2006).

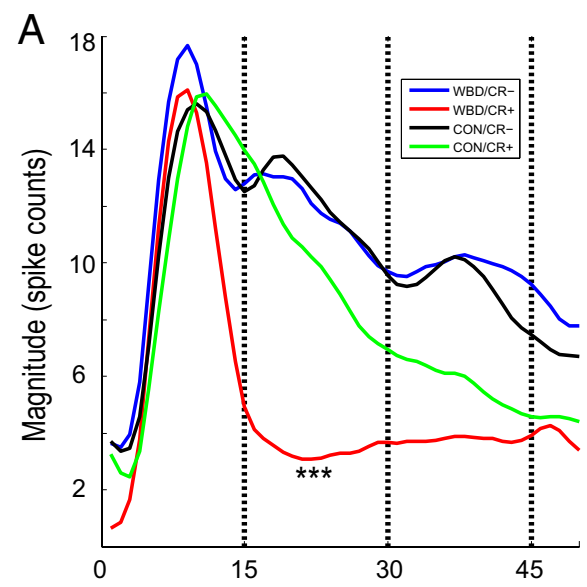

B

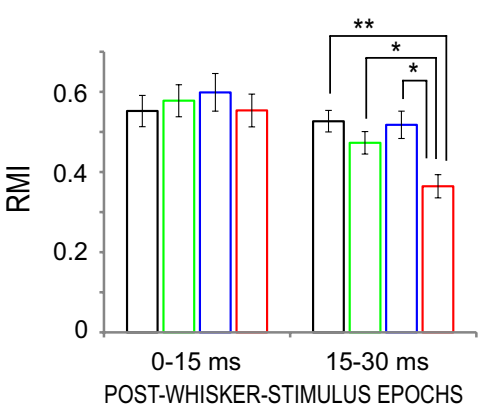

Figure 4. Population responses to the $C S$ stimulus and $\mathrm{RMl}$ in the different rearing groups showing the effect of rearing experience on cross-sensory responsiveness. $A$, Comparison of population PSTHs showing responses to the $C S$ stimulus for all four rearing groups during each $15 \mathrm{~ms}$ post-stimulus time bins (dashed, vertical, black lines) following the whisker stimulus. ${ }^{* *}$ Significant main effect for the 作 figure shows the raw ratios for ease of interpretation. The results were identical when comparisons were performed on the raw ratios

were observed in the CS condition). Second, because the index is a proportion we were able to estimate a grouped binomial mixed model that specified that the probability distribution of the residuals is binomial (Hilbe, 2009). The key feature of the analysis was the set of pairwise comparisons comparing $\mathrm{WBD} / \mathrm{CR}+$ to the other three groups on the RMI index. Dunnett's method (Dunnett, 1955) was used to adjust $p$ values. For the $0-15 \mathrm{~ms}$ epoch, there was no main effect for rearing group ( $p>0.05$ ), and the RMI for the WBD/CR+ failed to differ significantly from that of CON/CR $-(p>0.05)$, $\mathrm{CON} / \mathrm{CR}+(p>0.05)$, and WBD/CR $-(p>0.05)$ (Fig. $4 b)$. In contrast, for the 15-30 ms epoch there was a significant main effect for rearing groups $(p<0.01)$, and the RMI for WBD/CR+ was significantly different from CON/CR $-(p<0.01)$, CON/CR + $(p<0.05)$, and WBD/CR $+(p<0.05)$ (Fig. $4 b)$. Thus, the rearing experience significantly affected the RMI in the 15-30 ms time window, but not the early $0-15 \mathrm{~ms}$ epoch (Fig. 4).

\section{Discussion}

These results show that there is a significant cross-sensory auditory influence on the responses of S1 barrel cortex neurons in the normal adult rat that is modified by early sensory experience. 
There was a significant facilitation of whisker-evoked responses in the $0-15 \mathrm{~ms}$ time window, when an auditory click preceded a whisker stimulus, even though the barrel cortex neurons failed to fire action potentials in response to the auditory click stimulus alone. The response facilitation was similar across all rearing groups. In contrast, the response modulation during the CS stimulus in the longer latency 15-30 ms time window depended heavily upon postnatal rearing experiences. The conditioning auditory click before a whisker stimulus led to a modest but significant response facilitation in the normally reared CON/CRgroup that was especially robust in LIV and in low responsive neurons. A longer latency facilitation was observed only in the low responsive neurons after whisker trimming (WBD/CR-), while responses showed a strong tendency toward suppression in the low responsive neurons after click rearing $(\mathrm{CON} / \mathrm{CR}+)$. However, simultaneous whisker trimming coupled with click rearing $(\mathrm{WBD} / \mathrm{CR}+)$ produced a striking and significant response suppression in the 15-30 ms epoch following CS stimuli. This suppression was highly significant in both the supragranular and granular layers, for both FS and RS neurons, and for both low and high responsive neurons in the $\mathrm{WBD} / \mathrm{CR}+$ rats. Thus, auditory clicks profoundly modulate both the shorter $(0-15 \mathrm{~ms})$ and longer (15-30 ms) latency response epochs of barrel cortex neurons, while other combinations of rearing experiences influence primarily the longer latency cross-sensory responses. Finally, cross-sensory modulations may target subsets of neurons based on their response level, laminar location, and neuron type within each rearing condition.

The fact that the auditory clicks failed to drive any barrel cortex neurons under any conditions in the present study indicates that the cross-sensory multisensory interactions observed here are modulatory in nature (Dehner et al., 2004; Lakatos et al., 2007; Carriere et al., 2008), as opposed to the directly driven responses found in association cortex (Wallace et al., 1992; Carriere et al., 2007), and may account for the difficulty in detecting multisensory influences in primary sensory cortex. Surprisingly, the modulatory influence is present even in normally reared rats and is maximally enhanced when whisker deprivation is coupled with click rearing. Based on this, we propose that in all rearing groups the auditory click stimulation generates subthreshold changes in the excitability of barrel cortex neurons and, in that way, modulates the response firing rates of the neurons to their driving input (whiskers). This hypothesis requires testing with intracellular recording from the S1 neurons in response to auditory stimuli, and preliminary, unpublished intracellular recordings suggest that this indeed may be the case (M. Brecht, personal communication).

The modulatory cross-sensory influence was clearly detectable in the CS stimulus condition when the response magnitude was compared with whisker-only stimulus conditions (CS vs W1 or W2). The neuronal responses to W1, CS, and W2 were subdivided to separate the entire evoked-response period into time windows assumed to be dominated by thalamocortical inputs (0-15 ms) and a longer latency time window (15-30 ms) driven by strong corticocortical connections (Armstrong-James et al., 1992). One fundamental difference in the modulation of the two response components in the CS stimulus condition was that the putative thalamocortical responses were always facilitated in all four rearing groups, whereas the modulations in the corticocortical component differed significantly among the rearing conditions. For example, click-rearing conditions led to the CS stimulus reducing the probability of whisker-driven responses in the 15-30 ms epoch, with the effect being most significant in the
$\mathrm{WBD} / \mathrm{CR}+$ rats. Without click rearing, rats showed a tendency toward increased responses to CS stimulus in this epoch. This differential effect of rearing experience on the two poststimulus epochs suggests that the cross-sensory modulations in each epoch could be caused by different mechanisms. Thus, a two-stage model best fits the cross-sensory response modulations observed under our conditions (Fig. 5).

The response modulation observed in the $0-15$ ms epoch was always facilitative in nature. What is the best explanation for such facilitation in an early response period dominated primarily by thalamocortical inputs? There is ample evidence that subcortical auditory structures such as the dorsal cochlear nucleus (DCN) (Zhou and Shore, 2004; Haenggeli et al., 2005; Shore, 2005; Shore et al., 2008), the inferior colliculus (IC) (Jain and Shore, 2006; Zhou and Shore, 2006), and the medial division of the thalamic medial geniculate nucleus (mMGN) (Poggio and Mountcastle, 1960; Blum et al., 1979; Nicolelis et al., 1991) all receive significant somatosensory inputs and show multisensory responses. Thus, these structures, in turn, may influence subcortical somatosensory structures such as the trigeminal nucleus (TN) and the thalamic ventral posterior medial nucleus (VPM). One candidate structure that could mediate such cross-sensory interactions is the brainstem reticular formation (RF), which receives inputs from the DCN (Cant and Benson, 2003) and the IC (Kudo et al., 1983), and also projects directly to the TN (Ter Horst et al., 1991) and VPM (Bowsher, 1975). Thus, in the CS stimulus condition, when an auditory click is presented first, the subcortical auditory structures could activate the RF projections to subcortical somatic sensory structures to increase their excitability just before the whisker stimulus activates these structures. In addition, the cholinergic components of the RF could increase the excitability of VPM by disinhibiting inputs from the thalamic reticular nucleus (Berry et al., 1986; Lee et al., 1994a,b; Deschênes et al., 2005) (Fig. 5A-D, dashed line connections). Increased excitability of the VPM neurons would theoretically lead to increased activation by thalamocortical projections, which could explain the increase in spontaneous firing rates for non-click-reared rats and facilitation of response magnitude in the $0-15 \mathrm{~ms}$ for all groups of animals. This cross talk between subcortical sensory structures can thus be a probable mechanism for part of the early cross-sensory effect observed here, and is a testable hypothesis that was not directly addressed by the present experiments.

Although there were no significant changes in modulation between rearing groups in the $0-15 \mathrm{~ms}$ epoch, percentage analysis of individual neurons suggests that a higher percentage of neurons showed the facilitation in $\mathrm{CON}$ animals compared with the WBD animals and in the click-reared animals compared with the non-click-reared animals (e.g., CON/CR+ over WBD/CR+ and $\mathrm{CON} / \mathrm{CR}+$ over $\mathrm{CON} / \mathrm{CR}$ - rats). The difference between CON and WBD cortex may be due to the basic deficiency in the response magnitude of barrel cortex found after bilateral deprivation due to possible reduction in the synaptic efficacy of the thalamocortical inputs (Popescu and Ebner, 2010). The click rearing, in turn, might result in more effective, robust and widespread subcortical multisensory interactions and thus produce an increased percentage of neurons that show the facilitation in the 0-15 ms epoch.

The short latency facilitation is followed by modulation of responses in the 15-30 ms time window. There is little evidence in the literature for strong direct connections between A1 and S1 cortex in the rat that could provide the substrate for such a modulation. However, the dysgranular zone (DGZ) between A1 and 
A
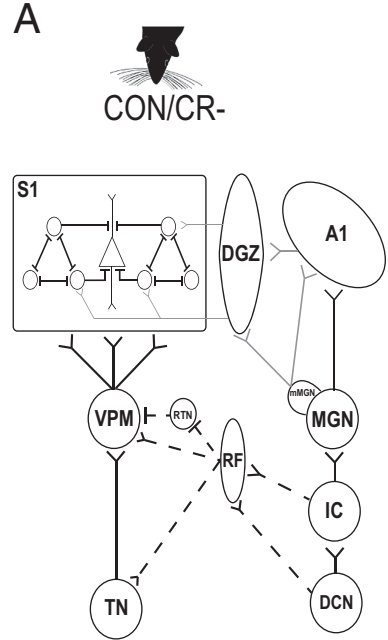

C

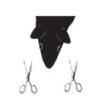

WBD/CR-
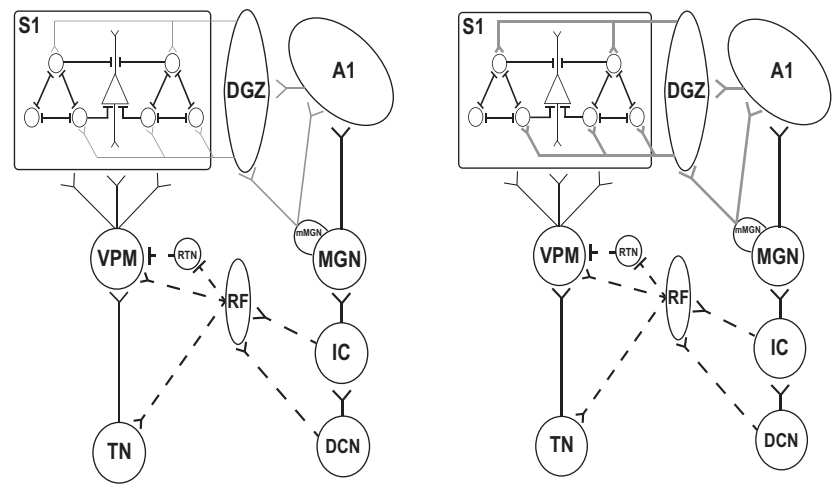

Figure 5. Hypothetical model proposing an explanation for the origin of the cross-sensory responses in $\$ 1$ barrel cortex. The mechanism of the cross-sensory responsiveness in S1 barrel cortex remains to be clarified. Here the four rearing conditions are shown with the auditory and somatic sensory relay structures projecting to $\mathrm{A} 1$ and $\mathrm{S} 1$ cortex labeled with the abbreviations listed below. The lines ending with an open fork indicate excitatory connections and those ending with a short bar indicate inhibitory connections. Dashed connections represent the possible anatomical substrates governing cross-sensory effects in the $0-15 \mathrm{~ms}$ epoch, and solid gray lines indicate connections for potential substrates of the 15-30 ms response epoch. Solid black connections highlight well known sensory pathways. Line thickness reflects the strength of a particular connection with thicker lines representing stronger connections and vice versa. Open circles and triangles in S1 symbolize FSUs and RSUs, respectively. According to this model, in all rearing groups the auditory subcortical structures facilitate activity in the somatic sensory subcortical structures via the $\mathrm{RF}$ (black dashed connections) and are responsible for the response facilitation observed in the $0-15 \mathrm{~ms}$ response epoch. On the other hand, the connections from the cortical DGZ that interconnect A1 and S1 cortex (gray connections) is postulated as the dynamic structure responsible for the response modulations observed in the 15-30 ms epoch. $A, D$, According to our hypothesis, the sparse and weak excitatory, auditory intracortical inputs onto inhibitory neurons in $\$ 1$ cortex in the $\mathrm{CON} / \mathrm{CR}$ - rats $(\boldsymbol{A})$ are upregulated with whisker deprivation and simultaneous click rearing $(\mathrm{WBD} / \mathrm{CR}+)$ both in their number and in synaptic efficacy $(\boldsymbol{D})$. This is thought to lead to the reported suppression observed in the 15-30 ms epoch of the $\mathrm{WBD} / \mathrm{CR}+$ rats. $\boldsymbol{B}, \boldsymbol{C}$, However, click rearing alone $(\boldsymbol{B})$ may produce stronger, but less numerous, DGZ-S1 connections to barrel cortex FSUs; whereas, bilateral whisker deprivation alone ( $C$ might lead to more numerous, but weak, corticocortical DGZ-S1 connections (gray lines) onto FSUs in barrel cortex. Both these effects in $\boldsymbol{B}$ and $\boldsymbol{C}$ would lead to a slightly higher level of inhibition in barrel cortex that could explain the absence of facilitation in the 15-30 ms epochs after CS stimulation under these rearing conditions (see text for detailed explanation of the model). RTN, Thalamic reticular nucleus. barrel cortex could mediate such an influence (Fig. $5 A-D$, gray connections). The DGZ neurons respond to both whisker and auditory stimuli (Brett-Green et al., 2003; Wallace et al., 2004). The DGZ receives auditory input from A1 cortex, from the ventral division of the MGN, and also from the multisensory neurons in the mMGN (Brett-Green et al., 2003). DGZ also projects directly to S1 barrel cortex (Chapin et al., 1987; Koralek et al., 1990). Moreover, since horizontal connections in cortex including somatosensory cortex have been shown to recruit inhibitory networks under some conditions (Tucker and Katz, 2003; Pluto et al., 2005; Keniston et al., 2010), it is possible that the projections from the DGZ to barrel cortex terminate on inhibitory FSUs that could cause subthreshold changes leading to an increased excitability in inhibitory neurons. Thus, this model predicts that the click component of the CS stimulus would activate the DGZ, which could increase the excitability of the FSUs in barrel cortex via corticocortical connections leading to a suppression of firing in the longer latency component of the barrel field responses. Moreover, since FSUs are interconnected to RSUs as well as each other, the inhibition should be observed in both cell types, which is consistent with our data.

The question remains open as to why there are significant rearing group differences in the response modulations of the $15-30 \mathrm{~ms}$ epoch. It is possible that with altered rearing experience that spans through the critical period the synaptic number, or efficacies, or both are modified for the DGZ to S1 connection. In normal animals, the facilitation observed in the $15-30 \mathrm{~ms}$ epoch was much smaller than that observed in the earlier $0-15 \mathrm{~ms}$ epoch. This reduction in facilitation could be due to active inhibition from the corticocortical connections by the mechanism described above. However, it is possible that the amount of inhibition is lower in normal animals because of sparse and lowefficacy synapses, and, thus, low-level facilitation is still observed in the 15-30 ms epoch (Fig. 5A). In rats that were whisker deprived (WBD/CR - ), the deprived state of the S1 cortex might lead to increased retention of the cross-sensory DGZ-S1 connections after maturation, without changing their synaptic efficacies from the normal levels. On the other hand, click rearing may increase the DGZ-S1 synaptic efficacies as opposed to the synaptic numbers. Any increase in inhibition due to the above two mechanisms could serve to reduce facilitation in the $15-30 \mathrm{~ms}$ response epoch of both the CON/CR + and the WBD/CR - rats (Fig. $5 B, C$ ). The dramatic suppression of the response in the 15-30 ms epoch in WBD/CR + rats could be a result of strong activation of numerous FSUs and consequently a steep increase in postexcitatory inhibition. By the above assumptions, this increased inhibition would be due to increase in both the synaptic density, possibly by whisker deprivation, and synaptic efficacy, due to simultaneous click rearing (Fig. 5D). The absence of increased spontaneous firing rate after the CS stimuli in the clickreared animals could be explained by an increase in the efficacy of such DGZ-S1 horizontal collaterals maintaining a relatively higher level of inhibition in click-reared barrel cortex. Finally, since the responses in the 15-30 ms epoch following the W2 stimulus remain significantly lower than those of W1 only in $\mathrm{WBD} / \mathrm{CR}+$ rats, it is possible that the CS stimulus has a longer acting effect on inhibition in this group of animals. It is also possible that the preceding set of auditory click stimuli alone (A2) could influence such a reduced response to W2.

The model described above is based on assumptions that can only be tested with further experiments. Recording from the subcortical structures under our conditions would be a key experiment that might shed light on whether the dual-mechanism 
hypothesis is tenable. An increase in excitability of neurons should be accompanied by an improved timing of response, and if the increase in inhibition is due to selective activation of FSUs, then there should also be an increase in neuronal synchrony and oscillations in barrel cortex after auditory stimuli (Cardin et al., 2009). This result would be similar to another study that showed that somatosensory stimulus-initiated oscillations reset the spontaneous oscillations in macaque A1 so that the phase of these reset oscillations produced enhanced auditory responses (Lakatos et al., 2007). Preliminary results suggest the click stimulus is indeed capable of changing the response timing properties of barrel cortex neurons, such as onset latency, spike synchrony, and spike coherence (Ghoshal et al., 2010).

Finally, although the model is consistent with our observed results, other mechanisms cannot be ruled out. For example, activity in the auditory pathways could influence the S1 barrel cortex via the nucleus basalis and/or amygdala (Gao and Suga, 1998; Ma and Suga, 2001). Further, the changes observed with altered rearing, especially with whisker deprivation, could be attributed to reported retention of direct projections from the mMGN to S1 barrel field (Nicolelis et al., 1991), although the latter study involved peripheral follicle damage (follicle cauterization) in contrast to the noninvasive whisker trimming used in these studies.

In conclusion, our results show that there is a significant auditory modulation of responses in the primary somatosensory cortex, and that this modulation is significantly altered by early postnatal rearing conditions, indicating that cross-sensory responsiveness is developmentally regulated by experience. In addition, the fact that such cross-sensory interactions can be detected even under anesthesia raises the possibility that crosssensory interactions in primary sensory cortex may be even more robust in awake, behaving rats and may facilitate the perceptual capability of the animals.

\section{References}

Armstrong-James M, Fox K, Das-Gupta A (1992) Flow of excitation within rat barrel cortex on striking a single vibrissa. J Neurophysiol 68:1345-1358.

Bavelier D, Neville HJ (2002) Cross-modal plasticity: where and how? Nat Rev Neurosci 3:443-452.

Berry DJ, Ohara PT, Jeffery G, Lieberman AR (1986) Are there connections between the thalamic reticular nucleus and the brainstem reticular formation? J Comp Neurol 243:347-362.

Blum PS, Abraham LD, Gilman S (1979) Vestibular, auditory, and somatic input to the posterior thalamus of the cat. Exp Brain Res 34:1-9.

Bowsher D (1975) Diencephalic projections from the midbrain reticular formation. Brain Res 95:211-220.

Brett-Green B, Fifková E, Larue DT, Winer JA, Barth DS (2003) A multisensory zone in rat parietotemporal cortex: intra- and extracellular physiology and thalamocortical connections. J Comp Neurol 460:223-237.

Brosch M, Selezneva E, Scheich H (2005) Nonauditory events of a behavioral procedure activate auditory cortex of highly trained monkeys. J Neurosci 25:6797-6806.

Burnett LR, Stein BE, Chaponis D, Wallace MT (2004) Superior colliculus lesions preferentially disrupt multisensory orientation. Neuroscience 124:535-547.

Cant NB, Benson CG (2003) Parallel auditory pathways: projection patterns of the different neuronal populations in the dorsal and ventral cochlear nuclei. Brain Res Bull 60:457-474.

Cardin JA, Carlén M, Meletis K, Knoblich U, Zhang F, Deisseroth K, Tsai LH, Moore CI (2009) Driving fast-spiking cells induces gamma rhythm and controls sensory responses. Nature 459:663-667.

Carriere BN, Royal DW, Perrault TJ, Morrison SP, Vaughan JW, Stein BE, Wallace MT (2007) Visual deprivation alters the development of cortical multisensory integration. J Neurophysiol 98:2858-2867.

Carriere BN, Royal DW, Wallace MT (2008) Spatial heterogeneity of corti- cal receptive fields and its impact on multisensory interactions. J Neurophysiol 99:2357-2368.

Chapin JK, Sadeq M, Guise JL (1987) Corticocortical connections within the primary somatosensory cortex of the rat. J Comp Neurol 263:326346.

Collignon O, Voss P, Lassonde M, Lepore F (2009) Cross-modal plasticity for the spatial processing of sounds in visually deprived subjects. Exp Brain Res 192:343-358.

Dehner LR, Keniston LP, Clemo HR, Meredith MA (2004) Cross-modal circuitry between auditory and somatosensory areas of the cat anterior ectosylvian sulcal cortex: a "new" inhibitory form of multisensory convergence. Cereb Cortex 14:387-403.

Deschênes M, Timofeeva E, Lavallée P, Dufresne C (2005) The vibrissal system as a model of thalamic operations. Prog Brain Res 149:31-40.

Dunnett CW (1955) A multiple comparison procedure for comparing several treatments with a control. J Am Stat Assoc 50:1096-1121.

Fitzmaurice GM, Laird NL, Ware JH (2004) Applied longitudinal analysis. New York: Wiley.

Foxe JJ, Schroeder CE (2005) The case for feedforward multisensory convergence during early cortical processing. Neuroreport 16:419-423.

Friedberg MH, Lee SM, Ebner FF (1999) Modulation of receptive field properties of thalamic somatosensory neurons by the depth of anesthesia. J Neurophysiol 81:2243-2252.

Gao E, Suga N (1998) Experience-dependent corticofugal adjustment of midbrain frequency map in bat auditory system. Proc Natl Acad Sci U S A 95:12663-12670.

Ghazanfar AA, Schroeder CE (2006) Is neocortex essentially multisensory? Trends Cogn Sci 10:278-285.

Ghoshal A, Pouget P, Popescu M, Ebner F (2009) Early bilateral sensory deprivation blocks the development of coincident discharge in rat barrel cortex. J Neurosci 29:2384-2392.

Ghoshal A, Nelson M, Pouget P, Ebner FF (2010) A novel mechanism for crossmodal interactions in normal rat $\mathrm{S} 1$ barrel cortex. In: Neuroscience 2010 meeting planner, Program No. 285.19. San Diego CA: Society for Neuroscience.

Guest S, Catmur C, Lloyd D, Spence C (2002) Audiotactile interactions in roughness perception. Exp Brain Res 146:161-171.

Haenggeli CA, Pongstaporn T, Doucet JR, Ryugo DK (2005) Projections from the spinal trigeminal nucleus to the cochlear nucleus in the rat. J Comp Neurol 484:191-205.

Hardin JW, Hilbe JM (2007) Generalized linear models and extensions, Ed 2. College Station, TX: Stata.

Hilbe JM (2007) Negative binomial regression. Cambridge, UK: Cambridge UP.

Hilbe JM (2009) Logistic regression models. London, UK: Chapman and Hall/CRC.

Jain R, Shore S (2006) External inferior colliculus integrates trigeminal and acoustic information: unit responses to trigeminal nucleus and acoustic stimulation in the guinea pig. Neurosci Lett 395:71-75.

Jousmäki V, Hari R (1998) Parchment-skin illusion: sound-biased touch. Curr Biol 8:R190.

Kayser C, Logothetis NK (2007) Do early sensory cortices integrate crossmodal information? Brain Struct Funct 212:121-132.

Keniston LP, Henderson SC, Meredith MA (2010) Neuroanatomical identification of crossmodal auditory inputs to interneurons in somatosensory cortex. Exp Brain Res 202:725-731.

Koralek KA, Olavarria J, Killackey HP (1990) Areal and laminar organization of corticocortical projections in the rat somatosensory cortex. J Comp Neurol 299:133-150.

Kral A (2007) Unimodal and cross-modal plasticity in the "deaf" auditory cortex. Int J Audiol 46:479-493.

Kudo M, Itoh K, Kawamura S, Mizuno N (1983) Direct projections to the pretectum and the midbrain reticular formation from auditory relay nuclei in the lower brainstem of the cat. Brain Res 288:13-19.

Lakatos P, Chen CM, O'Connell MN, Mills A, Schroeder CE (2007) Neuronal oscillations and multisensory interaction in primary auditory cortex. Neuron 53:279-292.

Lee SM, Friedberg MH, Ebner FF (1994a) The role of GABA-mediated inhibition in the rat ventral posterior medial thalamus. II. Differential effects of GABAA and GABAB receptor antagonists on responses of VPM neurons. J Neurophysiol 71:1716-1726.

Lee SM, Friedberg MH, Ebner FF (1994b) The role of GABA-mediated in- 
hibition in the rat ventral posterior medial thalamus. I. Assessment of receptive field changes following thalamic reticular nucleus lesions. J Neurophysiol 71:1702-1715.

Levin JR, Serlin RC, Seaman MA (1994) A controlled, powerful multiplecomparison strategy for several situations. Psychol Bull 115:153-159.

Li L, Rema V, Ebner FF (2005) Chronic suppression of activity in barrel field cortex downregulates sensory responses in contralateral barrel field cortex. J Neurophysiol 94:3342-3356.

Littell RC, Milliken GA, Stroup WW, Wolfinger, RD, Schabenberger O (2006) SAS for mixed models. Cary, NC: SAS Institute.

Ma X, Suga N (2001) Plasticity of bat's central auditory system evoked by focal electric stimulation of auditory and/or somatosensory cortices. J Neurophysiol 85:1078-1087.

Nicolelis MA, Chapin JK, Lin RC (1991) Neonatal whisker removal in rats stabilizes a transient projection from the auditory thalamus to the primary somatosensory cortex. Brain Res 567:133-139.

Pluto CP, Chiaia NL, Rhoades RW, Lane RD (2005) Reducing contralateral SI activity reveals hindlimb receptive fields in the SI forelimb-stump representation of neonatally amputated rats. J Neurophysiol 94:1727-1732.

Poggio GF, Mountcastle VB (1960) A study of the functional contributions of the lemniscal and spinothalamic systems to somatic sensibility. Central nervous mechanisms in pain. Bull Johns Hopkins Hosp 106:266-316.

Popescu MV, Ebner FF (2010) Neonatal sensory deprivation and the development of cortical function: unilateral and bilateral sensory deprivation result in different functional outcomes. J Neurophysiol 104:98-107.

Searle SR, Speed FM, Milliken GA (1980) Population marginal means in the linear model: an alternative to least squares means. Am Stat 34:216-221.

Shore SE (2005) Multisensory integration in the dorsal cochlear nucleus: unit responses to acoustic and trigeminal ganglion stimulation. Eur J Neurosci 21:3334-3348.

Shore SE, Koehler S, Oldakowski M, Hughes LF, Syed S (2008) Dorsal cochlear nucleus responses to somatosensory stimulation are enhanced after noise-induced hearing loss. Eur J Neurosci 27:155-168.
Simons DJ, Land PW (1987) Early experience of tactile stimulation influences organization of somatic sensory cortex. Nature 326:694-697.

Stein BE, Meredith MA 1990 Multisensory integration. Neural and behavioral solutions for dealing with stimuli from different sensory modalities. Ann N Y Acad Sci 608:51-65.

Stern EA, Maravall M, Svoboda K (2001) Rapid development and plasticity of layer 2/3 maps in rat barrel cortex in vivo. Neuron 31:305-315.

Ter Horst GJ, Copray JC, Liem RS, Van Willigen JD (1991) Projections from the rostral parvocellular reticular formation to pontine and medullary nuclei in the rat: involvement in autonomic regulation and orofacial motor control. Neuroscience 40:735-758.

Tucker TR, Katz LC (2003) Recruitment of local inhibitory networks by horizontal connections in layer $2 / 3$ of ferret visual cortex. J Neurophysiol 89:501-512.

Wallace MT, Meredith MA, Stein BE (1992) Integration of multiple sensory modalities in cat cortex. Exp Brain Res 91:484-488.

Wallace MT, Ramachandran R, Stein BE (2004) A revised view of sensory cortical parcellation. Proc Natl Acad Sci U S A 101:2167-2172.

Wiesel TN, Hubel DH (1965) Comparison of the effects of unilateral and bilateral eye closure on cortical unit responses in kittens. J Neurophysiol 28:1029-1040.

Zhang LI, Bao S, Merzenich MM (2002) Disruption of primary auditory cortex by synchronous auditory inputs during a critical period. Proc Natl Acad Sci U S A 99:2309-2314.

Zhou J, Shore S (2004) Projections from the trigeminal nuclear complex to the cochlear nuclei: a retrograde and anterograde tracing study in the guinea pig. J Neurosci Res 78:901-907.

Zhou J, Shore S (2006) Convergence of spinal trigeminal and cochlear nucleus projections in the inferior colliculus of the guinea pig. J Comp Neurol 495:100-112.

Zhou YD, Fuster JM (2000) Visuo-tactile cross-modal associations in cortical somatosensory cells. Proc Natl Acad Sci U S A 97:9777-9782.

Zhou YD, Fuster JM (2004) Somatosensory cell response to an auditory cue in a haptic memory task. Behav Brain Res 153:573-578. 